\title{
Long baseline experiments with LOFAR
}

\author{
Olaf Wucknitz ${ }^{* \dagger}$ \\ Argelander-Institut für Astronomie, Auf dem Hügel 71, 53121 Bonn, Germany \\ E-mail: wucknitzeastro.uni-bonn.de
}

I present first results of LOFAR observations with international baselines. An important cornerstone was the detection of the first long-baseline fringes. Their analysis turns out to be extremely useful to investigate and solve a number of technical issues of the instrument. Crude maps of the sky are created from single-baseline delay/fringe-rate spectra and compared with a short-baseline synthesis map. First long-baseline LBA images are shown of the source 3C196, a bright quasar whose sub-components can only be resolved with the long baselines. The corresponding subarcsec HBA image does not show the same amount of details yet, but HBA results are expected to improve significantly very soon. The LBA long-baseline image of 3C196 comprises the highestresolution radio map ever produced at this low frequency.

ISKAF2010 Science Meeting - ISKAF2010

June 10-14, 2010

Assen, the Netherlands

* Speaker.

$\dagger$ This work is supported by the Emmy-Noether-Programme of the 'Deutsche Forschungsgemeinschaft', reference WU 588/1-1, and by a Marie Curie European Reintegration Grant within the 7th European Community Framework Programme, Contract No. PERG02-GA-2007-224897 'WIDEMAP'. 


\section{Introduction}

The Low Frequency Array (LOFAR) will soon consist of 30-40 stations in the Netherlands plus international stations in Germany, France, England, Sweden and hopefully also Italy, Poland, Spain and other countries. Currently the only available international stations are all in Germany: Effelsberg near Bonn, Tautenburg near Jena, Unterweilenbach near Munich and Bornim near Potsdam.

LOFAR will offer wide fields of view, observations with several independent beams (in different directions) and a high survey speed. Two subsystems cover a frequency range between about 10 and $250 \mathrm{MHz}$. The Low Band Antennas (LBA) are used for $10-80 \mathrm{MHz}$ (only 30-80 will be used regularly because of strong RFI at lower frequencies), and the High Band Antennas (HBA) for $110-250 \mathrm{MHz}$. The FM band is excluded intentionally.

On long baselines, sub-arcsec resolution will be possible even in the LBA band, which opens an entirely new parameter space for radio observations. Because of the high number of stations, full synthesis imaging is possible.

\section{Resolution}

The main motivation for international baselines is the possibility to reach much higher resolutions than with Dutch stations alone. One exciting application of LOFAR surveys is the search for gravitational lenses [3]. These objects can be identified directly from the survey data provided that sub-arcsec resolution can be achieved. The reliability of this identification and thus the efficiency of lens surveys depends strongly on the resolution and the sensitivity on long baselines. The resolution that can be achieved with LOFAR is shown in Tab. 1 as a function of frequency and baseline length.

\begin{tabular}{|c|c|c|c|c|c|}
\hline freq/MHz & $\lambda / \mathrm{m}$ & $1 \mathrm{~km}$ & $30 \mathrm{~km}$ & $300 \mathrm{~km}$ & $1000 \mathrm{~km}$ \\
\hline 10 & 30 & 1.7 & $3 ! 4$ & $21^{\prime \prime}$ & $6 . " 2$ \\
\hline 30 & 10 & $34^{\prime}$ & $1 ! 1$ & 6."9 & $2 . " 1$ \\
\hline 80 & 3.8 & $13^{\prime}$ & $26^{\prime \prime}$ & $2 . " 6$ & $0 . " 77$ \\
\hline 120 & 2.5 & 8.6 & $17^{\prime \prime}$ & 1.7 & $0 . " 52$ \\
\hline 160 & 1.9 & 6.4 & $13^{\prime \prime}$ & $1 . " 3$ & $0 . " 39$ \\
\hline 220 & 1.5 & 4.7 & $9 . " 4$ & 0.'94 & $0 .{ }^{\prime \prime} 28$ \\
\hline
\end{tabular}

Table 1: Fringe-spacing $\theta=\lambda / L$ as estimate for the resolution achievable with LOFAR for different combinations of frequency and baseline length. Sub-arcsec resolution requires baselines of at least several hundred (HBA) or thousand (LBA) km length.

\section{International stations available for this work}

For the observations described here, only the international stations in Effelsberg, Tautenburg and Unterweilenbach were used (see Fig. 1). Bornim was still waiting for its network connection, and stations in other countries were not constructed yet. 

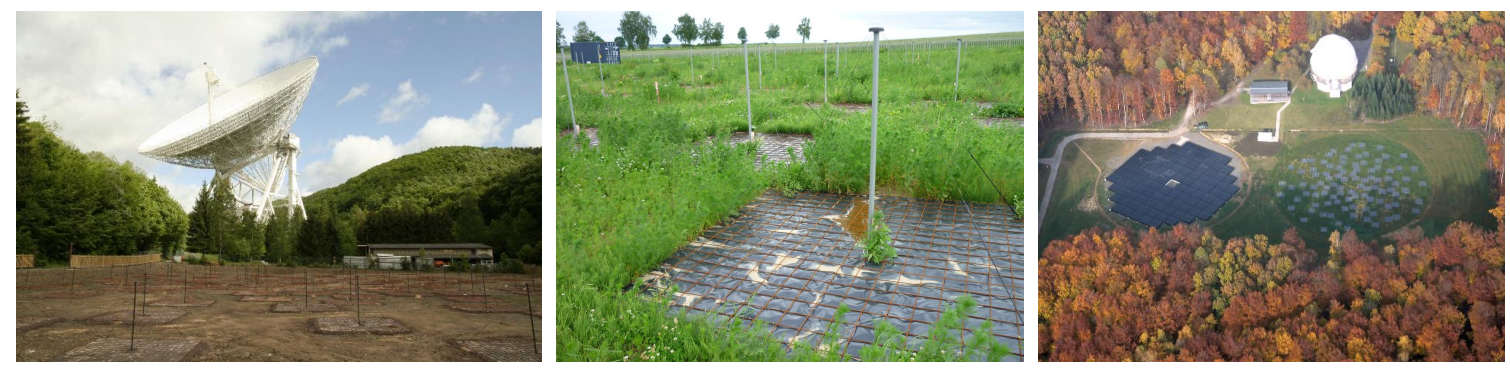

Figure 1: Left: LBA field of the Effelsberg station with the $100 \mathrm{~m}$ radio telescope in the background, centre: LBA antennas in Unterweilenbach, right: the Tautenburg station with HBA and LBA fields and the dome of the $2 \mathrm{~m}$ optical telescope.
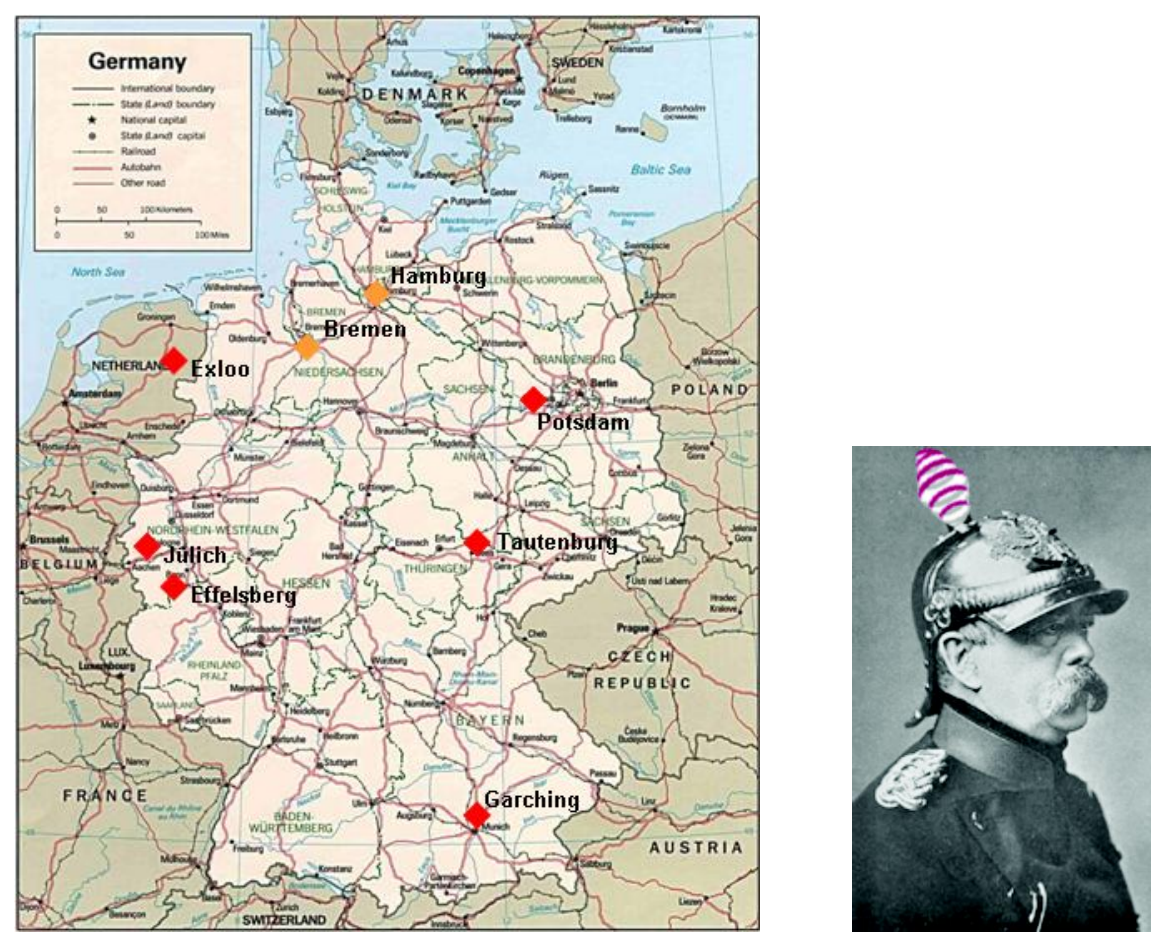

Figure 2: Location of LOFAR stations in Germany (image courtesy of AIP) and the inofficial logo of the German subarray ('Otto-von-Bismarck-Telescope', invented by a colleague from Dwingeloo who is not known to be of German persuasion).

The baseline lengths between the Dutch core and German stations are about $300 \mathrm{~km}$ (Effelsberg), $400 \mathrm{~km}$ (Tautenburg) and $600 \mathrm{~km}$ (Unterweilenbach), compare Fig. 2.

\section{Long baseline issues}

A major issue on long baselines is the fact that the majority of sources will at least be partially resolved so that the signal weakens considerably on longer baselines. This is also true for calibrator sources so that the number of good calibrators is reduced.

In order to achieve a sufficient signal-to-noise ratio $(S / N)$ not only for imaging, but in the first instance for the calibration, the data have to be (implicitly or explicitly) averaged in time 
and frequency. In order to do that, the dependence of phases on time and frequency has to be taken into account. Delays (proportional to derivatives of phase with respect to frequency) and rates (derivatives of phase with respect to time) have to be fitted and corrected for. In Very Long Baseline Interferometry (VLBI), this technique is known as fringe-fitting and is applied on a regular basis.

In the context of LOFAR, with the very low frequencies and very large fractional bandwidth, the procedure is considerably more complicated than in standard VLBI observations, because one has to distinguish between non-dispersive and dispersive delays. Non-dispersive delays ( $\tau$ independent of $v$ ) are due to clock offsets and errors in positions of stations and sources, while dispersive delays $\left(\tau \propto v^{-2}\right)$ are caused by the ionosphere. Generally it turns out that both are important. We find that the clock offsets can be much larger than the ionospheric delays (sometimes many $\mu$ sec when the clock synchronisation fails), but they tend to be constant with time and are thus easier to correct. Ionospheric delays of several $100 \mathrm{nsec}$ are often observed on long baselines at frequencies around $50 \mathrm{MHz}$. Both effects have to be taken into account when averaging over several subbands.

At the moment, fringe-fitting is only partly implemented in own software. I can fit both kinds of delays on individual baselines. Because the dispersion is not significant over a single subband, FFTs can be used within the subbands. Interpolated results of that are combined coherently over all subbands to determine multi-band coherent delay solutions.

With the wide bands, the rates have to be treated as delay rates instead of phase rates, because the latter would be frequency dependent both for dispersive and non-dispersive delays. The software thus fits four parameters (plus a phase) for blocks of data that typically extend over 10-60 sec in time and up to $48 \mathrm{MHz}$ in frequency.

In addition it is found, not unexpectedly, that differential Faraday rotation between the stations is generally significant at lower frequencies and on long baselines. This means that the XX and YY correlations are not sufficient to determine Stokes I, because parts of the signal are shifted to the mixed correlations XY and YX. Finally the imaging on long baselines is much more difficult than for the Dutch array alone, because the $u v$ coverage is much more sparse.
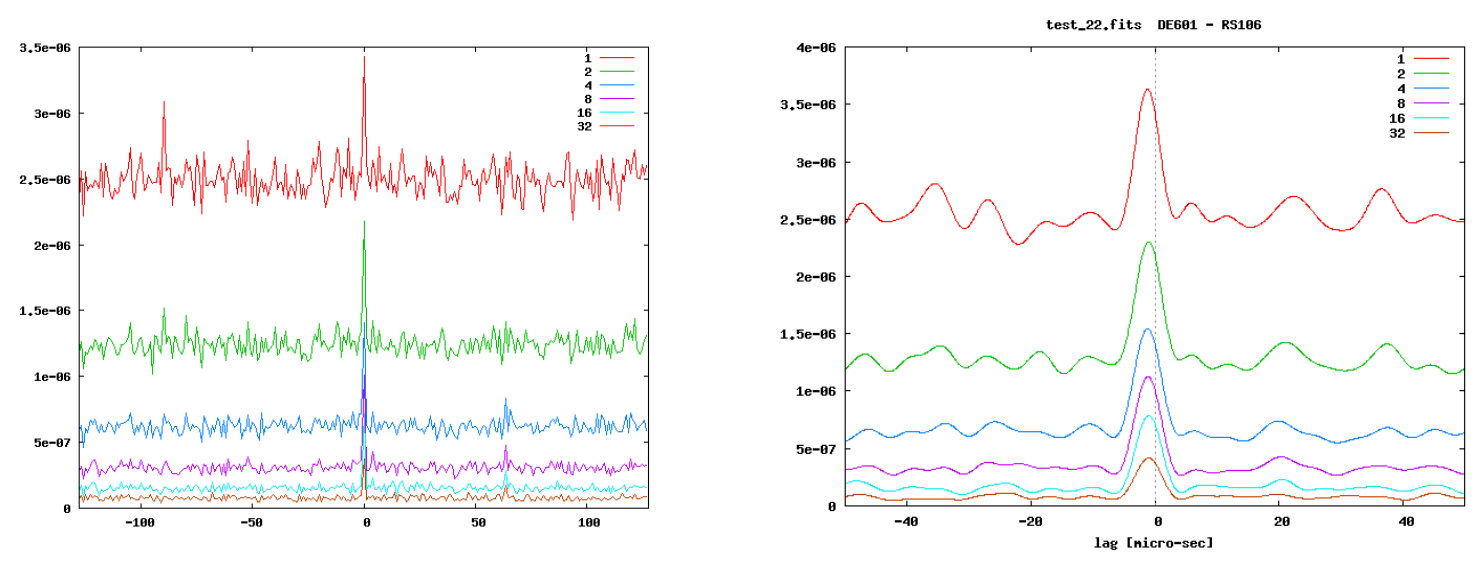

Figure 3: Delay spectra on a baseline between the Netherlands and Effelsberg. The different colours denote different coherent averaging times (labels in units of the integration time of $5 \mathrm{sec}$ ). The second plot with higher delay resolution shows that the peak is slightly offset from 0. 


\section{First long-baseline fringes}

Shortly after others had seen hints of short periods of consistent phases on LBA observations of 3C196 that included the station Effelsberg, I started a more systematic search for fringes in the parameter space of delay and rate. For the first attempt in August 2009, data of one subband were coherently averaged over short intervals, then Fourier transformed on the frequency axis to obtain delay spectra, and the power was averaged (incoherently) over one hour to increase the $S / N$. Results are shown in Fig. 3.

The clear peak in the delay spectra near a lag of 0 did not convince everybody at that time, because other effects may mimic real fringes caused by a sky signal, particularly if the peak is at exactly zero delay. Plotting the spectra with higher resolution revealed that the peak is in fact offset by about one $\mu$ sec.

A more systematic analysis followed in which the time-dependence was explicitly taken into account by scanning a two-dimensional (and finally four-dimensional) parameter space of delay and rate. See Fig. 4 for the first single-band and multi-band results, again for observations of 3C196.
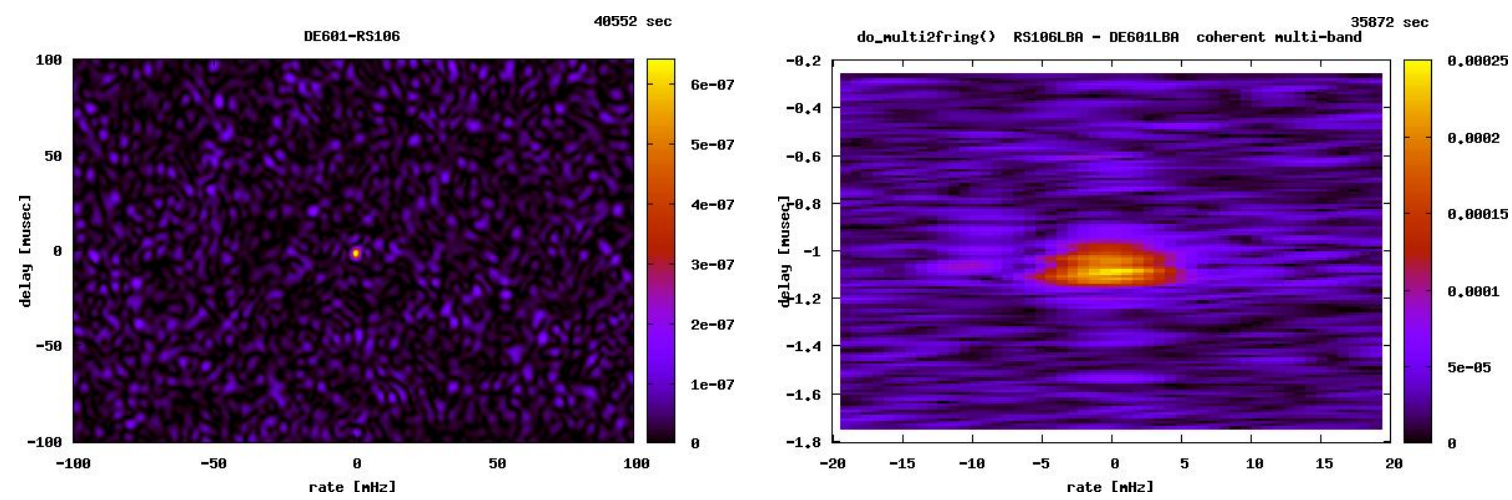

Figure 4: Fringe-rate/delay spectra for a short period of observations of 3C196 on a baseline between the Netherlands and Effelsberg. Left: single-band with low resolution on the vertical delay axis, right: coherent combination of many bands with much higher resolution in delay. The strange shape of the peak is partly caused by not including dispersive effects in this plot. Animated versions are available at http://www.astro.uni-bonn.de/ wucknitz/download/ 2009_dwingeloo_lofar_status_fring4a.gif and http://www.astro.uni-bonn.de/ wucknitz/download/2009_dwingeloo_lofar_status_msfring1_coh.gif.

This analysis started on the short baselines, where the strongest fringes were surprisingly found for delays and rates far from zero and of the same order of magnitude as the total geometric delays and rates. It was first thought that the delay tracking did not work properly, but then I found that the stronger fringes are coming from other bright sources like Cygnus A or Cassiopeia A. Their signal, leaking in through high-order sidelobes of the station beam, can be stronger than the target signal even if they are more than $90^{\circ}$ away from the station beam centre.

The next two German stations coming online were Tautenburg and Unterweilenbach with first LBA observations in January 2010. Shortly thereafter I detected fringes to Tautenburg, then to Unterweilenbach and finally even between pairs of German stations. The first fringes between Tautenburg and Unterweilenbach are shown in Fig. 5. 


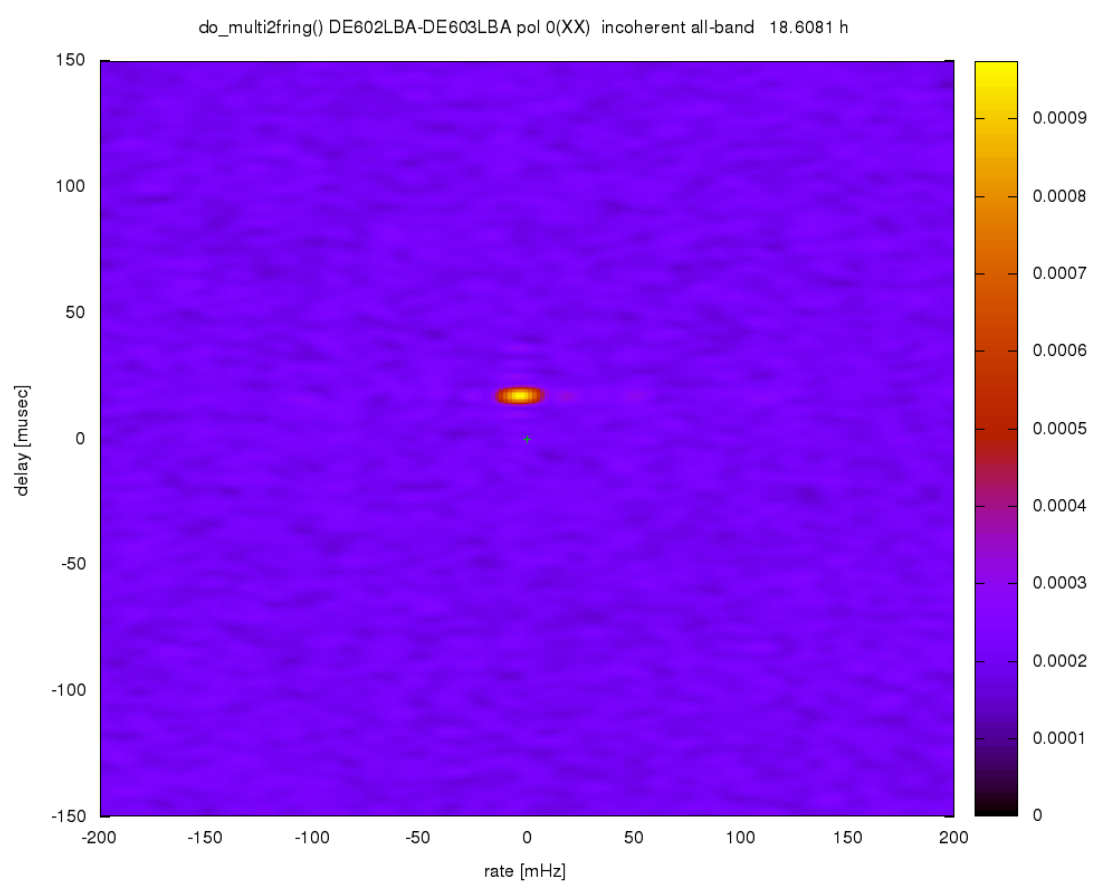

Figure 5: First fringes on the baseline Tautenburg-Unterweilenbach (January 2010). The delay of about $17 \mu \mathrm{sec}$ is caused by a clock offset in the Tautenburg station that resulted from an incorrect station position in the GPS configuration. This problem was fixed later. In the movie (http://www . astro. uni-bonn. de/ wucknitz/download/2010_feb17_lofar_status_anim.mp4) we sometimes see other peaks moving around slowly. They are caused by other sources in the field.

Applying the elementary geometry of the Earth rotation, I transformed delay/rate values to sky position and in this way (after averaging over a short period) produced a delay/fringe-rate map of the field around 3C196 using data from only one baseline (see Fig. 6). Besides the target 3C196, three other sources can be seen more or less clearly.

For comparison, a wide-field synthesis map was produced using only Dutch baselines. Fig. 7 shows the result and a VLSS map of the same field.

\section{Results from fringe-analysis}

The most important result is the clear detection of fringes in long-baseline LBA observations. In addition a number of technical issues were found in the course of this analysis. Among them are the relatively large clock offsets in German stations, some confusion about the labelling of LBA polarisations under certain circumstances, a strong $8 \mathrm{MHz}$ ripple that resulted from accidentally disabling the cable-length compensation in the beam forming, the detection of strong differential Faraday rotation and an independent detection of a resonance near $63 \mathrm{MHz}$.

Most of these problems have been fixed later. The independent long-baseline analysis contributed significantly to that process, even though this was not the primary intention of my efforts. 
DE601LBA-DE602LBA pol O(XX) incoherent multi-band

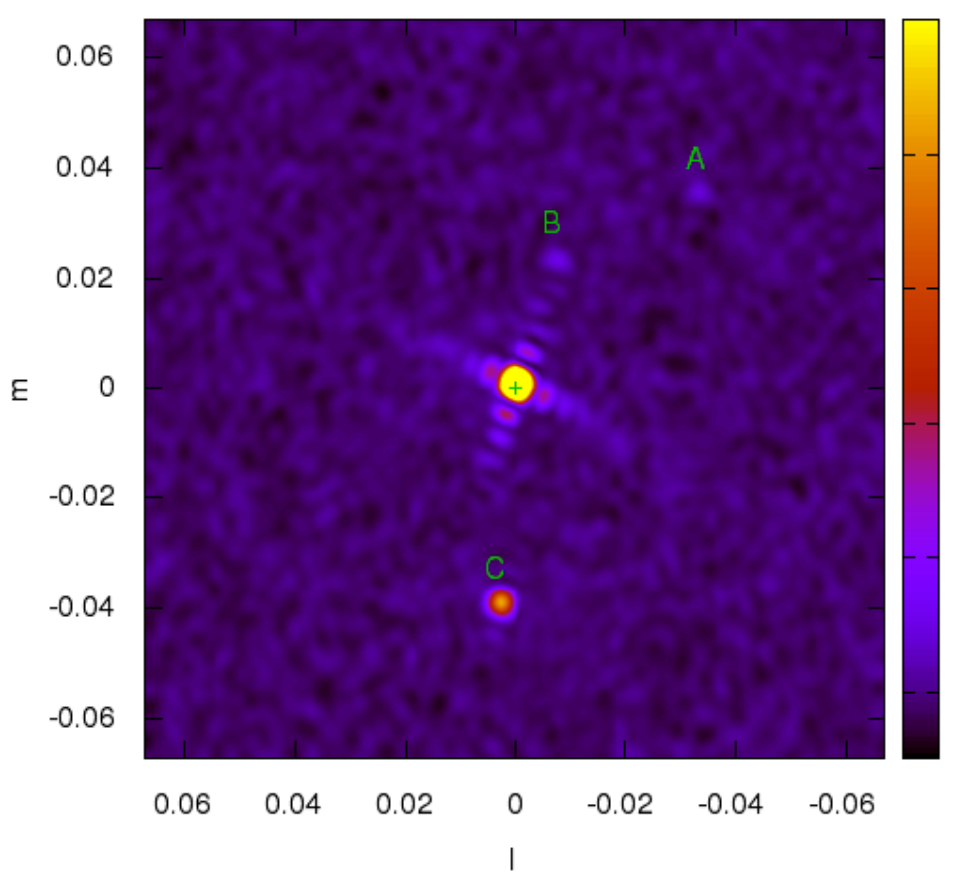

Figure 6: Delay/fringe-rate map around 3C196 created from delay/rate spectra of the long baseline, Effelsberg-Unterweilenbach. The bright source in the centre is 3C196 with about $140 \mathrm{Jy}$. The other three sources are listed in the VLSS with fluxes of 19/6/17 Jy for A/B/C at 74 MHz, respectively. Coordinates are $l, m$ in radians, 0.04 corresponds to about 2.3 . This map is also the JIVE/ASTRON picture of the day of 3 rd March 2010.

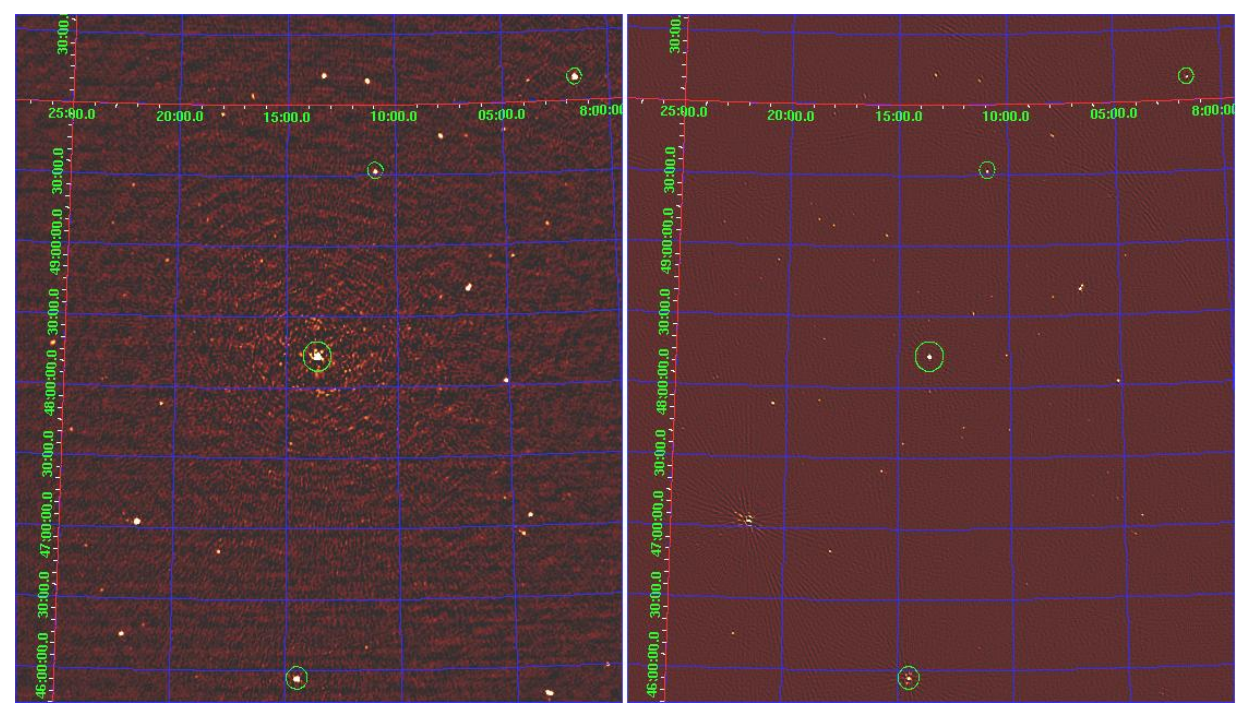

Figure 7: VLSS map (left) and LOFAR LBA map (right) of the field around 3C196. Only Dutch baselines were used in the imaging. 3C196 in the centre was used as the (only) calibrator. The three sources A/B/C from Fig. 6 and 3C196 in the centre are marked with circles. We see that, as expected, the calibration is much better close to the centre of the field than further away. 


\section{First long-baseline maps: LBA}

For the first serious long-baseline imaging attempt, a $6 \mathrm{~h}$ observation on the target 3C196 on 12/13th Feb 2010 (data set D2010_16704) was used. Five Dutch and three German stations (Effelsberg, Unterweilenbach and Tautenburg) produced a wide range of baselines lengths. Only a fraction of the 160 subbands (30-80 MHz with small gaps) showed a strong signal because of the aforementioned $8 \mathrm{MHz}$ ripple. In the first attempts, only the good subbands were used, later the weaker ones were included, too.

In the analysis I first corrected for the $1 \mu \mathrm{sec}$ and $17 \mu \mathrm{sec}$ clock offsets in Effelsberg and Tautenburg, applied some flagging, then converted the XX/XY/YX/YY polarisation combinations to circular RR/RL/LR/LL in order to avoid differential Faraday rotation, averaged somewhat in time and frequency, and then finally imaged and self-calibrated RR and LL in AIPS and difmap. For the final maps produced with difmap, Gaussian components with variable spectral indices were fitted to the data in order to correct the MFS (multi frequency synthesis) imaging for spectral index variations.
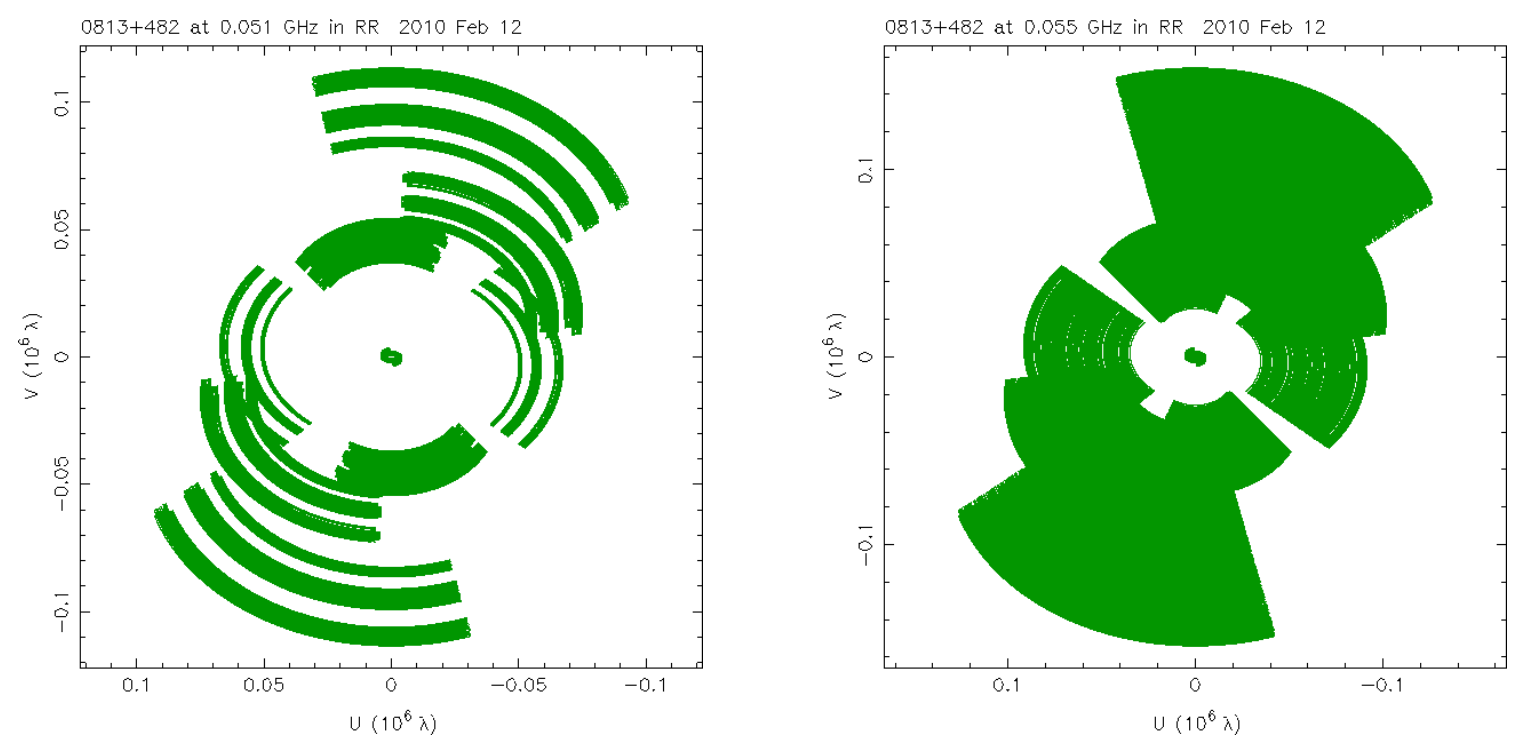

Figure 8: $u v$ coverage of the long-baseline LBA observations. Left: only the 'good' subbands, right: the entire frequency range. The Dutch baselines form the compact small region in the centre. All other baselines are combinations of/with German stations.

The $u v$ coverage is shown in Fig. 8. The expected appearance of the source and the very first LOFAR images are shown in Fig. 9. To my surprise, about four instead of the expected two components showed up in the images. However, the inspection of $408 \mathrm{MHz}$ MTRLI (MERLIN) observations revealed a very similar structure (see Fig. 11).

The comparison with the MERLIN images gave more confidence in the reliability of the imaged structures. The process was repeated and improved to produce the currently best long-baseline LBA LOFAR image shown in Fig. 10. The direct comparison with the $408 \mathrm{MHz}$ image is shown in Fig. 11.

These maps have the highest resolution that has ever been achieved in this frequency range so far. They are a great step forward for low-frequency VLBI. 

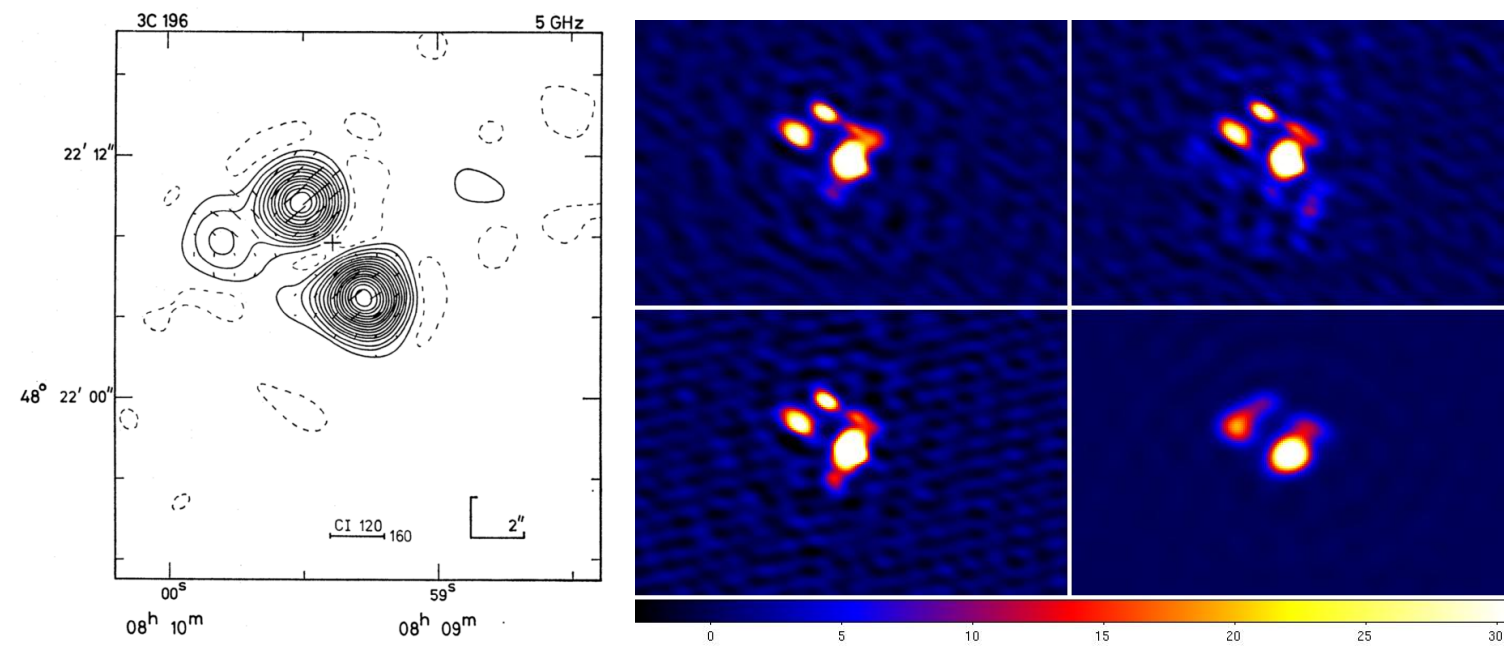

Figure 9: Left: $3 \mathrm{C} 196$ at $5 \mathrm{GHz}$ observed with the Cambridge $5 \mathrm{~km}$ array [from 2], component separation ca. 5."5, right: four early attempts to image the same source from LOFAR LBA data using slightly different calibration strategies. All four panels show basically the same structures. The lower right panel was produced form a different data set with only one German station (Effelsberg). In this case the long-baseline information came from the amplitudes alone, because no closure phases are available with effectively only one long baseline. The amplitudes were stable during the observation.

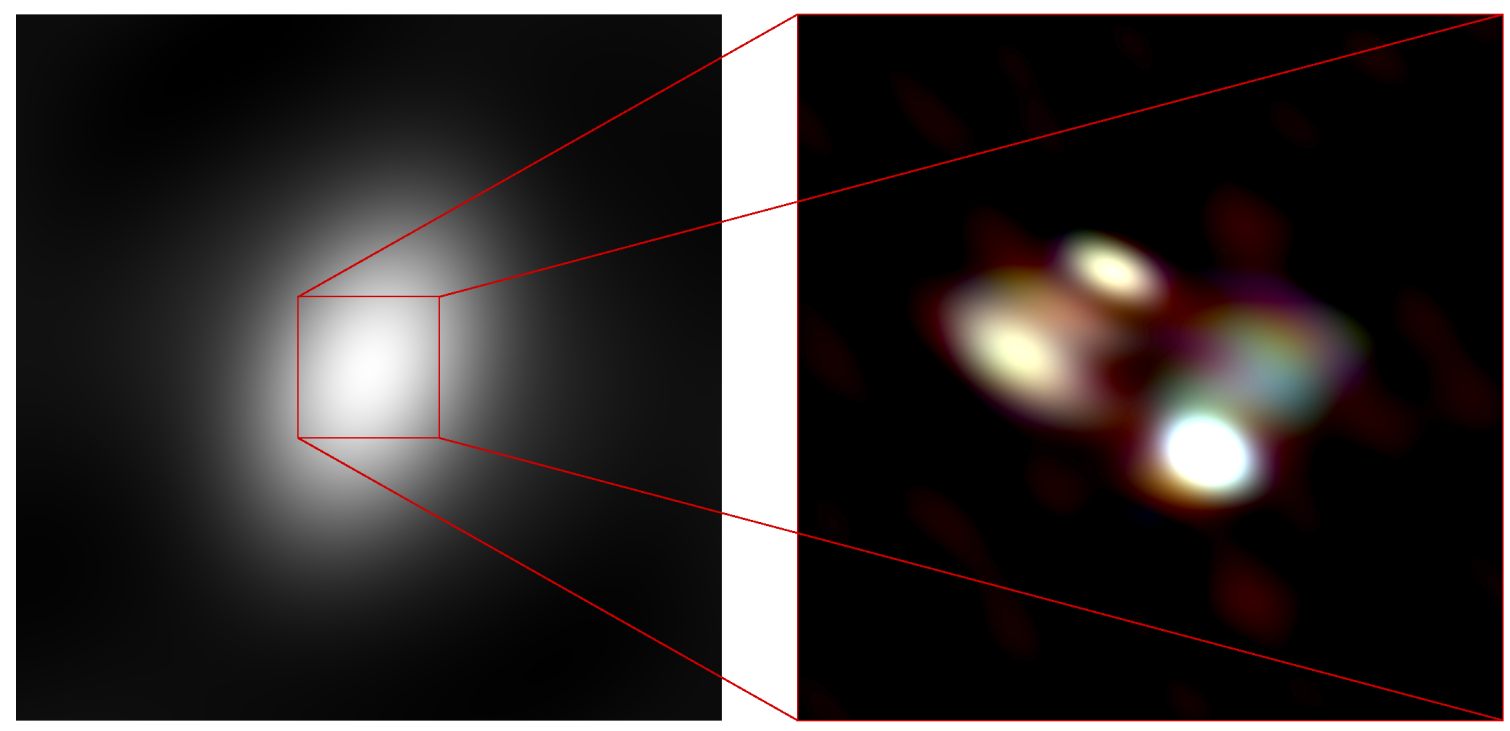

Figure 10: Left: Map of $3 \mathrm{C} 196$ using only the Dutch baselines. The resolution of $35^{\prime \prime} \times 22^{\prime \prime}$ is not sufficient to resolve any details. Right: Blowup using all the long baselines. With a resolution of $11^{\prime \prime} 5 \times 00^{\prime \prime} 9$, the structures of the target are finally revealed. The colours are chosen to resemble what the human eye would see if it were sensitive to radiation at a wavelength ten million times larger than visible light. This is also the JIVE/ASTRON picture of the day of 1st June 2010. 


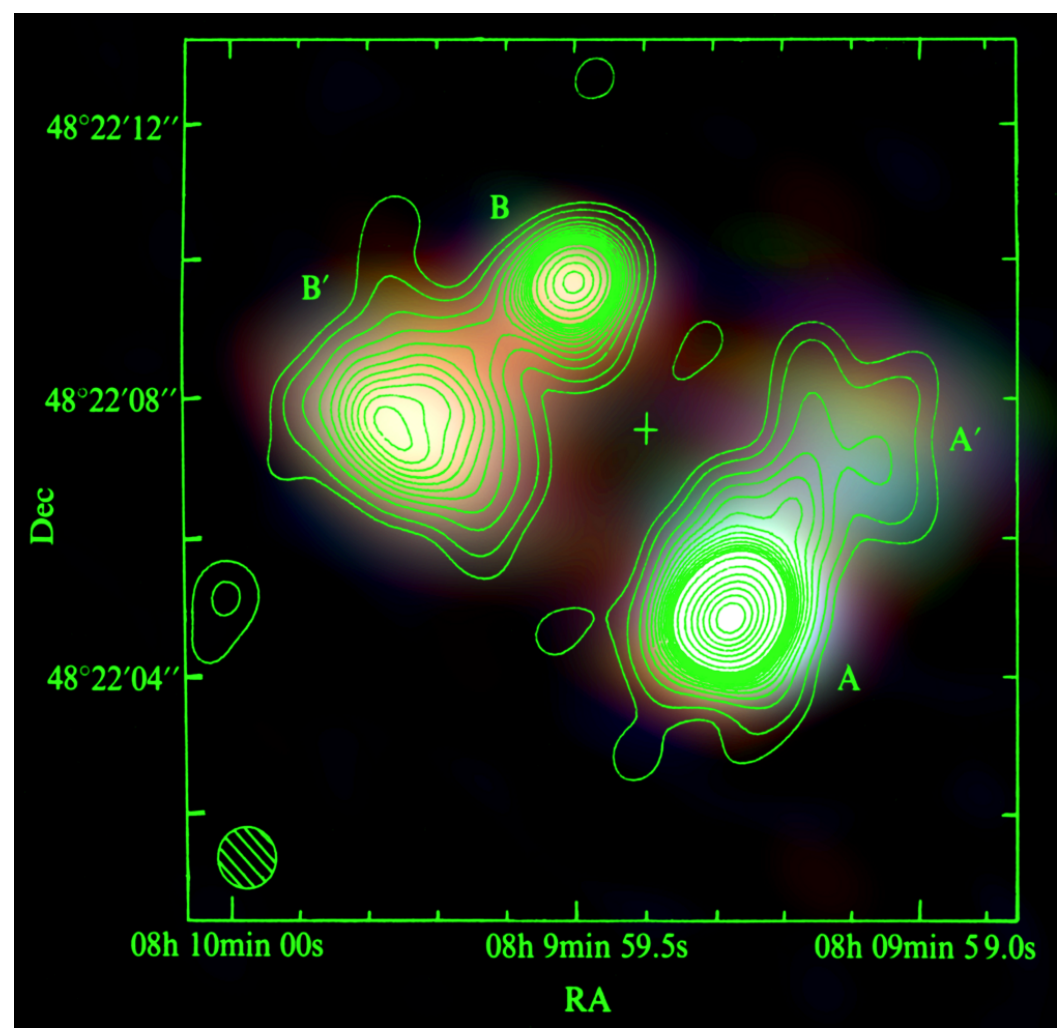

Figure 11: Comparison of the LOFAR image of $3 \mathrm{C} 196$ (background image) with the $408 \mathrm{MHz}$ observations (contours from Lonsdale \& Morison [1]). The structures match up surprisingly well, given the factor of 8 in frequency. Note that the self-calibration process started conservatively with a point-source model and continued without prior knowledge of the $408 \mathrm{MHz}$ details.

\section{First long-baseline maps: HBA}

Progress with the HBA is still lagging behind. Shortly after the first long-baseline HBA observations, fringes could be confirmed, but they turned out to be much weaker than expected. The imaging was correspondingly intricate and has not produced satisfying results so far.

For the first image, observation L2010_07608 of 3C196 was used (12 h on 22nd May 2010). The frequency range was $131-155 \mathrm{MHz}$ in 120 subbands. 7 Dutch and only two German stations (Effelsberg and Tautenburg) were used. I had to correct for the clock offset in Effelsberg and for an offset of $8 \mu \mathrm{sec}$ in the superterp stations in the core in order to allow averaging in frequency. The imaging and self-calibration was done in difmap and used the YY correlation. Most of the time the $S / N$ on the long baselines was very low, particularly on the baseline Effelsberg-Tautenburg. The reason for this is not fully understood. Partially it is due to the source structure, but more important seems to be the (then still missing) station calibration. Some inconsistencies between blocks of subbands made the averaging in frequency difficult.

The $u v$ coverage and dirty beam are shown in Fig. 12, the very preliminary map in Fig. 13. So far we only see compact parts of three of the components at HBA frequencies. 

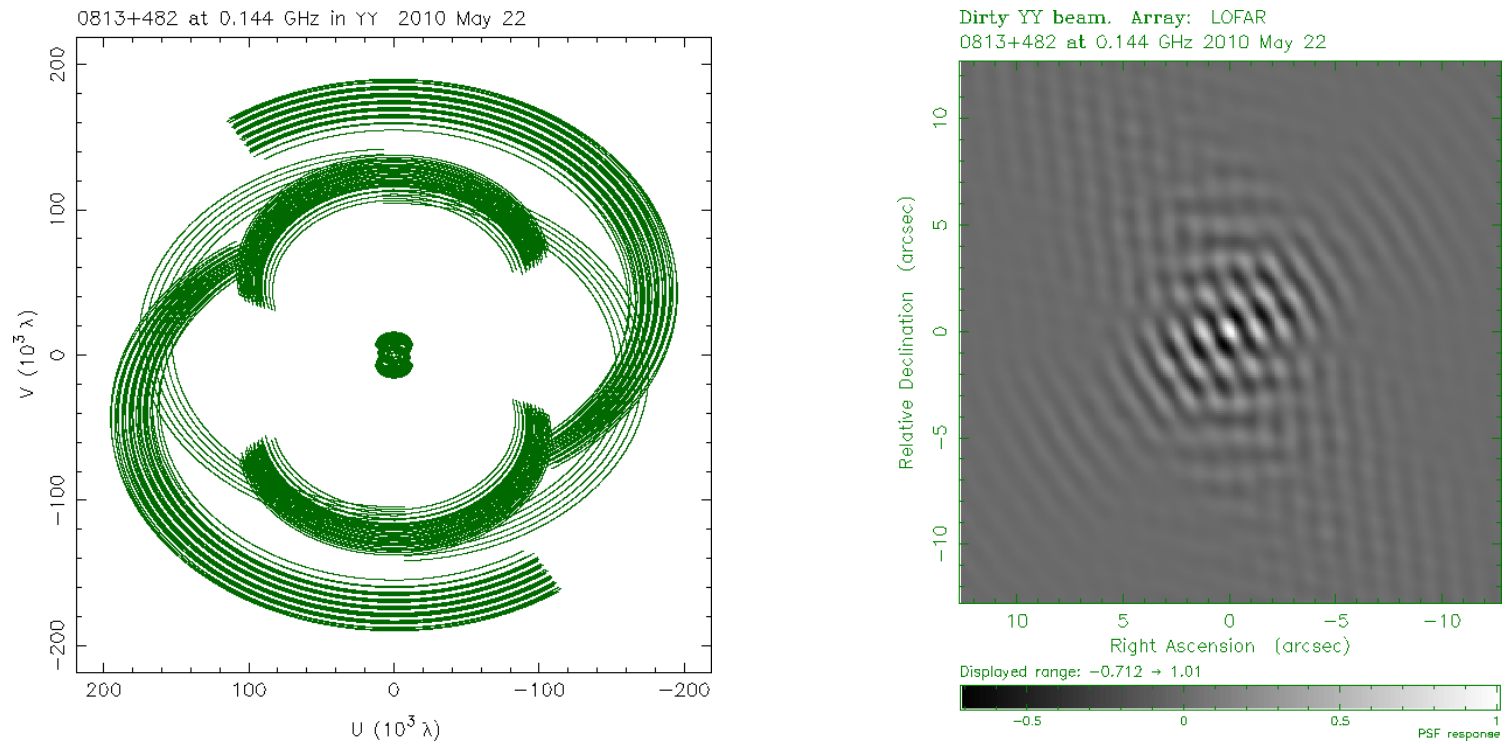

Figure 12: Left: $u v$ coverage, right: calibrated dirty beam of the HBA observations of $3 \mathrm{C} 196$. The strong sidelobes make the imaging process very unstable and difficult.
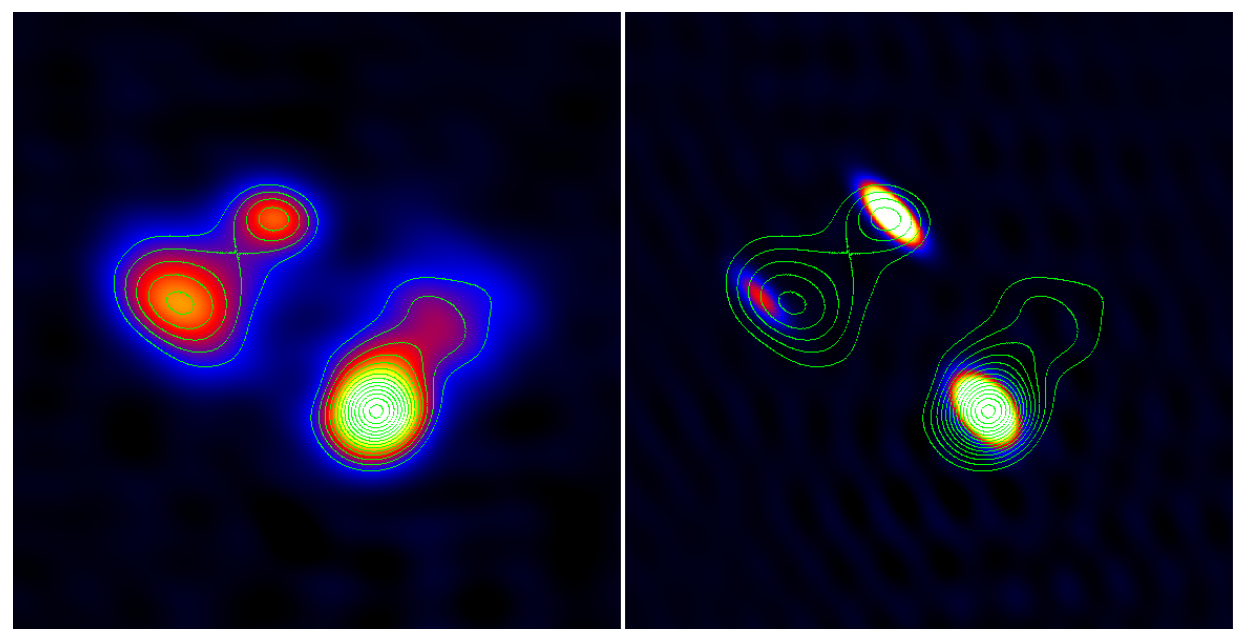

Figure 13: Left: LBA map described before, right: preliminary HBA map with superposed LBA contours. Even though the details are not $100 \%$ reliable yet, it looks as if we only see the brightest and most compact regions in three of the components. They do not coincide with the centroids of the LBA emission. This is not entirely surprising, because all components seen are lobes with unresolved hot spots. The core of the AGN is not seen at radio wavelengths.

\section{Acknowledgements}

I would like to thank many colleagues in the LOFAR team whose work made these results possible. In particular the contributions of the following people were directly related to the results presented here. From ASTRON: Ashish Asgekar, Michiel Brentjens, André Gunst, George Heald, John McKean, Menno Norden, Antonis Polatidis and Stefan Wijnholds. From the German stations: James Anderson (Effelsberg), Annette Haas and Matthias Hoeft (Tautenburg). 


\section{Summary}

This contribution proves that the long baselines of LOFAR actually work. They produce fringes, they can be calibrated, and they can be used to produce high-resolution images of the low-frequency radio sky. The highest-resolution image in the frequency range $30-80 \mathrm{MHz}$ has been produced from only three international (plus five Dutch) stations, a small part of the final array. Fringe-fitting methods are required to determine and correct dispersive and non-dispersive delays in order to allow averaging in frequency. Phase changes with time turn out to be not much worse on long baselines than on short ones. Beyond about $30 \mathrm{~km}$, the ionospheric patches are more or less independent of each other, so that variations do not increase with baseline length anymore. Even though the delays are larger, they can be calibrated because of their stability.

In order to analyse these data, new analysis techniques had to be developed and implemented. The results presented here were achieved with software written particularly for this purpose together with the standard packages AIPS and di fmap.

The original presentation of the conference talk together with a number of fringe movies is available from the author's homepage at http: / www . astro. uni-bonn. de/ wucknitz/ publications/pub.php?2010_iskaf_assen_lofar.

\section{References}

[1] Lonsdale, C. J. \& Morison, I.: 1980, Rotationally symmetric structure in two extragalactic radio sources, Nature 288, 66-69.

[2] Pooley, G. G. \& Henbest, S. N.: 1974, Observations of 48 extragalactic radio sources with the Cambridge 5-km telescope at $5 \mathrm{GHz}$, MNRAS 169, 477-526.

[3] Wucknitz, O. \& Garrett, M.: 2008, Gravitational lens surveys with LOFAR, Proceedings of the conference From Planets to Dark Energy: the Modern Radio Universe, PoS (MRU) 142. 